\title{
Absorbing boundaries in numerical solutions of the time-dependent Schrödinger equation on a grid using exterior complex scaling
}

\author{
F. He, C. Ruiz, A. Becker \\ Max-Planck-Institut für Physik of komplexer Systeme, Nöthnitzer Str.38, D-01187 Dresden, Germany
}

(Dated: August 23, 2021)

\begin{abstract}
We study the suppression of reflections in the numerical simulation of the time-dependent Schrödinger equation for strong-field problems on a grid using exterior complex scaling (ECS) as absorbing boundary condition. It is shown that the ECS method can be applied in both the length and the velocity gauge as long as appropriate restrictions are applied in the ECS transformation of the electron-field coupling. It is found that the ECS method improves the suppression of reflection as compared to the conventional masking technique in typical simulations of atoms exposed to an intense laser pulse. Finally, we demonstrate the advantage of the ECS technique to avoid unphysical artifacts in the evaluation of high harmonic spectra.
\end{abstract}

PACS numbers: $32.80 . \mathrm{Rm}, 33.80 . \mathrm{Rv}, 42.50 . \mathrm{Hz}$

\section{INTRODUCTION}

With the progress in laser technology in recent years [1, 2], the focused laser field strengths increased rapidly to exceed the strength of the Coulomb fields that bind the electrons in the ground state of an atom or molecule. Such intense light fields have led to the discovery of novel aspects of light-matter interactions, such as multiphoton ionization, above-threshold ionization, high harmonic generation, multiple ionization to high charge states, Coulomb explosion etc. The lowest-order perturbation theory of light-matter interaction is known to break down at light intensities above about $I \simeq 5 \times 10^{12}$ $\mathrm{W} / \mathrm{cm}^{2}$ for near optical wavelengths [3]. Several nonperturbative theories of laser-matter interaction, such as the numerical solution of the time-dependent Schrödinger equation (TDSE) [4, 5, 6, 7], ab-initio methods based on the Floquet theorem [8, 9, 10], basic set expansion methods [11, 12, 13] or $S$-matrix and related theories [14, 15, 16, 17], have been developed. Among these the solution of the TDSE on a time-space grid is considered as a rigorous and powerful approach. For the investigation of most of the above-mentioned intense-field phenomena the simulation of an atom with $N$ electrons, which would require the solution of a set of $3 \times N$ dimensional partial differential equations, can be well approximated using the single-active-electron (SAE) model [4]. In the latter only one of the electrons of the atom is considered to become active and to interact with the external field, which reduces the numerical problem to at most three dimensions. Any symmetry of the external field may further reduce the dimensionality.

Accurate solutions of time-dependent problems on the grid require not only relatively dense grid points but, in general, also a huge spatial extension of the grid to account for the release and the motion of the electron sub-wave packets in the field. These factors result in large memory and CPU requirements for the numerical solution at high field intensities. Fortunately, the important dynamics of several intense-field processes occur on a spatial volume close to the atom or molecule. A few examples are the determination of ionization rates [4] and high harmonic spectra [5] or the identification of dominant pathways to single [18] or nonsequential double ionization [19] of a molecule. These studies can be therefore performed on a relatively small grid neglecting the exact form of the outgoing ionizing parts of the wave function. It however requires to suppress reflections from the edges of the numerical grid, which can cause artificial effects, e.g. in the form of spurious harmonics in high harmonic spectra [5].

In calculations based on the numerical propagation of wave packets in different areas of physics and chemistry several techniques have been proposed to compensate for reflections, including masking functions (or equivalently absorbing imaginary potentials) [5, 20, 21], repetitive projection and Siegert state expansion methods [22], complex coordinate rotation or exterior complex scaling 23, 24] and others. In the numerical solution of the time-dependent Schrödinger equation of atoms in intense laser fields masking functions or absorbing potentials are the most commonly used techniques (e.g. 5, 25, 26, 27, 28, 29]). The ECS technique is rarely used in this context up to now 32, 33, 34, which might be due to the fact, that its application in the electric field (or length) gauge has been thought to be not fruitful [33].

In this paper we re-examine the implementation of the exterior complex scaling (ECS) method [30, 31] as absorbing boundary condition in simulations of strong-field problems. In particular, we focus on the application of the ECS technique in different gauges, namely the length and the velocity gauge. On the basis of results of simulations of 1D model atom exposed to an oscillating external field, we investigate appropriate restrictions of the ECS transformation of the external-field coupling to avoid undesired effects in the absorbing area. Results for the probability density and momentum distributions for the interaction of the hydrogen atom with an intense laser field are then compared with those obtained using the standard masking function technique. Finally, we consider a typical strong-field problem by using the ECS method to calculate high harmonic spectra. 


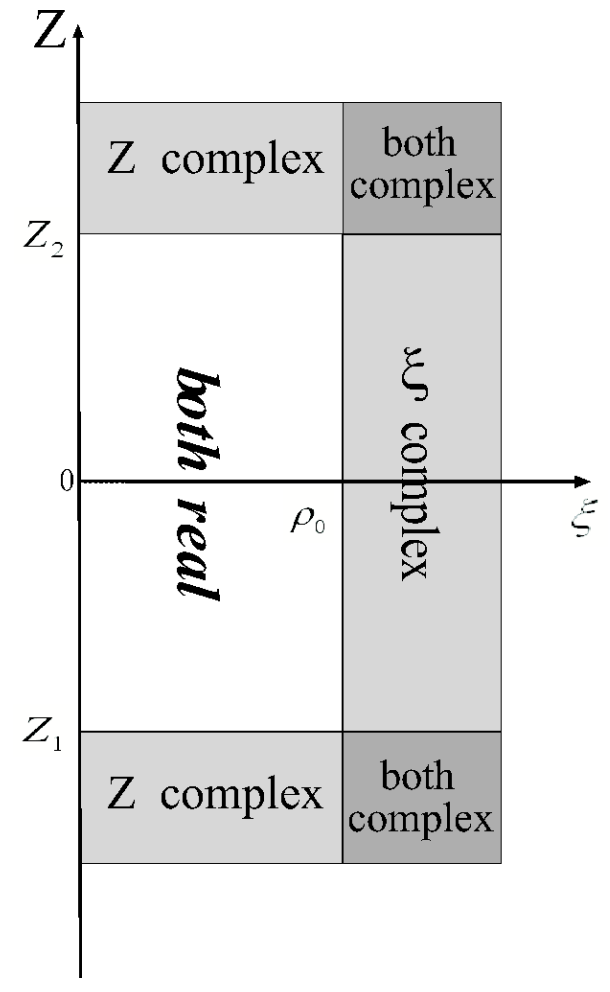

FIG. 1: Scheme of the ECS coordinate transformation. Real coordinates are used in the interior box, defined by $z_{1} \leq z \leq$ $z_{2}$ and $\rho \leq \rho_{0}$. The zones in gray mark the areas, where one or both coordinates are complex.

\section{EXTERIOR COMPLEX SCALING (ECS) AS ABSORBING BOUNDARY}

The complex scaling method has been widely used in physics and chemistry (for a review, see [39]), e.g. in the theory of potential scattering 40], calculation of resonances in atoms and molecules [41] or the calculation of cross sections in scattering processes 42. According to this method the radial coordinate of the particles are scaled by a complex phase factor, which distorts the spectrum of the Hamiltonian such that the continuous spectrum is rotated in the complex energy plane and the discrete resonance eigenvalues are revealed. For our aim to introduce absorbing boundaries at the edges of a numerical grid in time-dependent simulations we make use of an extension of the complex scaling method, namely the exterior complex scaling (ECS) technique [31], in which the spatial coordinates are only scaled outside some distance from the origin.

As discussed at the outset the ECS technique has been used before [32, 33, 34] in time-dependent studies of electron impact ionization as well as of the motion of a charged particle in dc and ac electromagnetic fields. In the latter context it has been mentioned [33] that it appears to be not fruitful to apply ECS in the electric field (or length) gauge. We now revisit this question and analyze below how ECS can be used in both the length and the velocity gauges in simulations of atoms exposed to intense laser pulses.

\section{A. Implementation}

First, we illustrate the implementation of the ECS technique in $1 \mathrm{D}$ and $2 \mathrm{D}$ time-dependent calculations, it is straightforward to extend it to higher dimensions (c.f. [24, 33]. Let us consider the non-relativistic electron dynamics in a time-independent (Coulomb) potential $V_{0}$ and an external field governed by the time-dependent Schrödinger equation in cylinder coordinates as (Hartree atomic units, $m_{e}=\hbar=e=1$, are used throughout):

$$
i \frac{\partial}{\partial t} \psi(z, \rho ; t)=\left[H_{0}(z, \rho)+V(t)\right] \psi(z, \rho ; t)
$$

with the time-indepentent Hamiltonian

$$
H_{0}(z, \rho)=-\frac{1}{2} \frac{\partial^{2}}{\partial \rho^{2}}-\frac{1}{2 \rho} \frac{\partial}{\partial \rho}-\frac{1}{2} \frac{\partial^{2}}{\partial z^{2}}+V_{0}(z, \rho)
$$

and time-dependent external-field coupling, given in length or velocity gauge, as

$$
V(t)= \begin{cases}z E(t), & \text { length gauge } \\ \frac{A}{c} \hat{p}_{z}, & \text { velocity gauge }\end{cases}
$$

Here $E(t)$ is the electric field and $A(t)$ is the vector potential of the external electromagnetic pulse linearly polarized along the $z$-direction.

The ECS transformation on the two coordinates $z$ and $\rho$ can be given by (c.f. Fig. 1):

$$
\begin{aligned}
& Z= \begin{cases}z_{1}+\left(z-z_{1}\right) \exp (i \eta) & \text { as } z<z_{1} \\
z & \text { as } z_{1} \leq z \leq z_{2} \\
z_{2}+\left(z-z_{2}\right) \exp (i \eta), & \text { as } z>z_{2}\end{cases} \\
& \xi= \begin{cases}\rho & \text { as } \rho \leq \rho_{0} \\
\rho_{0}+\left(\rho-\rho_{0}\right) \exp (i \eta), & \text { as } \rho>\rho_{0}\end{cases}
\end{aligned}
$$

where $\eta$ is the scaling angle with $0<\eta<\pi / 2$. $z_{1}, z_{2}$ and $\rho_{0}$ are labeled in Fig. 1 and define the size of the interior box $\left(z_{1} \leq z \leq z_{2}\right.$ and $\left.\rho \leq \rho_{0}\right)$ within which both spatial coordinates are real. Outside (gray zones in Fig. 1) one or both coordinates are complex.

It is the aim of the ECS method to transform the outgoing wave into a function, which falls off exponentially outside the interior box, while the wave-function keeps unchanged in the region where the coordinates are real [24, 36, 37]. In case of the present problem of an atom exposed to an oscillating linearly polarized electromagnetic pulse, we therefore investigate whether or not the transformed solution of Eq. 1 shows this desired behavior. To this end and without loss of generality, we restrict our analysis to the $Z$ direction, i.e. the direction of the external field. The time-dependent solution of the 1D analogous of Eq. 1 in the complex area can be written as:

$$
\psi(t+\Delta t) \sim \exp \left[-i\left(H_{0}(Z)+V(t)\right) \Delta t\right] \psi(t)
$$


The time-independent operator in Eq. (6) is given by:

$$
\begin{aligned}
& \exp \left[-i H_{0}(Z) \Delta t\right]= \\
& \quad \exp \left[-i \frac{\cos (2 \eta)}{2} \hat{p}_{z}^{2}\right] \exp \left[-\frac{\sin (2 \eta)}{2} \hat{p}_{z}^{2}\right] \\
& \quad \times \exp \left[-i \operatorname{Re}\left(V_{0}\right) \Delta t\right] \exp \left[\operatorname{Im}\left(V_{0}\right) \Delta t\right]
\end{aligned}
$$

As discussed by McCurdy et al. 33] the exponent in the second factor on the right hand side of Eq. (7) is always negative if $0<\eta<\pi / 2$ and provides already the desired decay term. It is therefore important to note that the wave-function will be basically absorbed in the complex area due to the transformed kinetic operator term as long as there are no counteracting effects from other terms in the Hamiltonian. In general, it is therefore required that $\operatorname{Im}\left(V_{0}\right) \leq 0$ such that the last term in Eq. (7) acts as an absorbing potential. In practice, the discontinuity in the real and imaginary part of the potential terms introduced by the complex scaling factor can generate some small reflections. An efficient way to avoid this numerical problem appears to keep the $Z$-coordinate in the potential term untransformed as a real number in the absorption area. Please note that it is unproblematic to abandon the additional absorption effect of the imaginary potential, since the decay of the wavefunction is already ensured via the kinetic operator term.

The time-dependent potential in Eq. (6) can be written as

$$
\begin{aligned}
\exp [ & -i E(t) Z \Delta t] \\
= & \exp \left[-i E(t)\left(z_{1,2}+\left(z-z_{1,2}\right) \exp (i \eta)\right) \Delta t\right] \\
= & \exp \left[E(t)\left(z-z_{1,2}\right) \sin \eta \Delta t\right] \\
& \times \exp \left[-i E(t)\left(z_{1,2}+\left(z-z_{1,2}\right) \cos \eta\right) \Delta t\right]
\end{aligned}
$$

in the length gauge or as

$$
\begin{aligned}
& \exp \left[-i\left(\frac{A(t)}{c} \exp (-i \eta) \hat{p}_{z}\right) \Delta t\right] \\
& =\exp \left[-\sin \eta \frac{A(t)}{c} \hat{p}_{z} \Delta t\right] \exp \left[-i \cos \eta \frac{A(t)}{c} \hat{p}_{z} \Delta t\right](9)
\end{aligned}
$$

in the velocity gauge. In the right sides of Eqs. (8) and (9), the second factors are oscillatory ones, and are simple scaled versions of the external-field coupling as the wavefunction enters the complex area. The first factors, however, can act both as an absorber or as an undesired source, depending on the sign of the exponent. It is determined in the length gauge by the instantaneous sign of the oscillating electric field, while in the velocity gauge, it equals the sign of the product $A(t) \hat{p}_{z}$. Next, we will analyze the effects of the oscillating field coupling term in numerical simulations and show that an undesired explosion of the wavefunction can be avoided by using the standard untransformed coupling even in the absorption region.
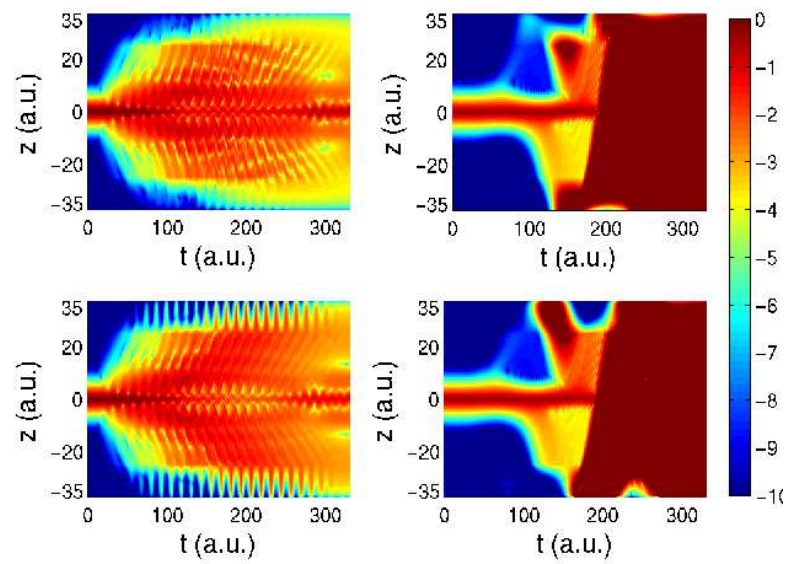

FIG. 2: (color online) Temporal evolution of the probability density from the numerical simulation of a 1D model atom interacting with a high-frequency field (left hand column) and a Ti:sapphire field (right hand column). Shown is a comparison of the results obtained using the external field coupling in the velocity gauge (upper row) and in the length gauge (lower row).

\section{B. ECS technique in the length and the velocity gauges}

In order to investigate the effects of the oscillating field on the transformed wavefunction we have performed 1D test calculations with a soft atomic model potential

$$
V_{0}=-\frac{1}{\sqrt{1+z^{2}}}
$$

using two different external fields, namely a highfrequency electric field given by

$$
E= \begin{cases}E_{1} \sin \left(\omega_{1} t\right) t / 5 T, & \text { as } t \leq 5 T \\ E_{1} \sin \omega_{1} t, & \text { as } t>5 T\end{cases}
$$

where $E_{1}=0.5, \omega_{1}=0.5$ and $T=2 \pi / \omega_{1}$, and a 3 -cycle low-frequency Ti:sapphire laser pulse

$$
E=E_{2} \cos \left(\omega_{2} t\right) \sin ^{2}\left(\pi t / L_{2}\right)
$$

with $E_{2}=0.1$ a.u., $\omega_{2}=0.057$ a.u. and $L=330$ a.u.. The respective vector potentials are derived from the electric field expressions in Eqs. (11) and (12). Please note that the former field, which is smoothly turned on over 5 optical cycles, is similar to the field form used by McCurdy et al. 33]. The real part of the calculation box is restricted in both cases by $z_{1}=-z_{2}=-25$ a.u., with the complex part extending over 12.5 a.u. on both sides of the grid.

In Fig. 2 we present the temporal evolution of the electron density distributions in the high-frequency field (left hand column) and in the Ti:sapphire field (right hand column). The panels in the upper and the lower row show the numerical results obtained in the length 

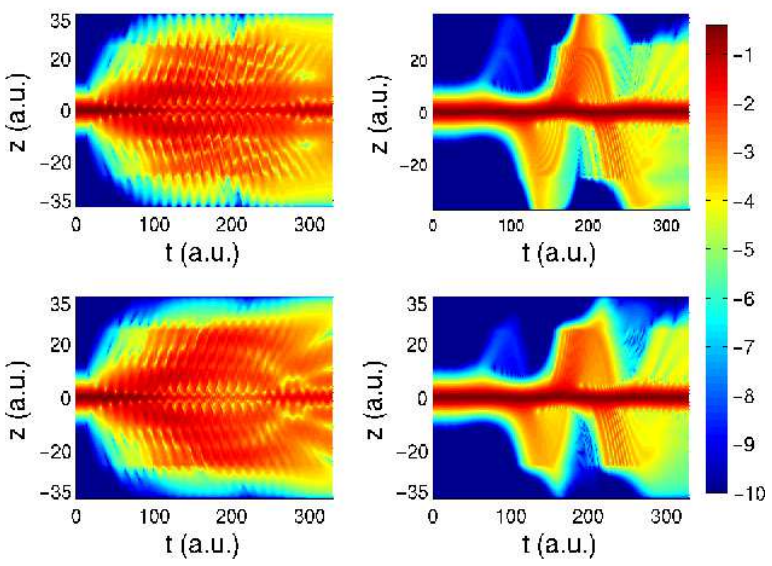

FIG. 3: (color online) Same as Fig. 2 but using the untransformed standard field coupling on the entire grid.

and the velocity gauge, respectively. The effect of the first factor in Eqs. (8) and (9) is most clearly seen in the results of the low-frequency calculations, where we observe an explosion of the wave-funtion in the second part of the evolution after a significant part of the wavefunction has entered the complex area. This is obviously due to the fact that the term acts as a source over a half cycle of the pulse. As expected above the same unphysical feature is found in the length as well as in the velocity gauge. In the high-frequency case (left-hand column) the results obtained in the two gauges are again similar, but we do not observe a significant amount of explosion. This difference as compared to the Ti:sapphire calculations is probably due to two factors: First, in the high-frequency simulation the probability density, which enters the complex area, is smaller than in the low-frequency calculations. Second, the rapid change of the sign of the electric field or the vector potential may effectively prevent an explosion, since the complex factor quickly changes between decay and source nature.

From the results presented above we may therefore infer that the ECS transformation may lead to unphysical results in both gauges due to the oscillating nature of the external field. The most straightforward strategy to circumvent this problem appears to simply neglect the ECS transformation of the coordinates in the field coupling term. This means in other words that the untransformed standard field coupling (c.f. Eq. (3) ) is used over the entire grid including the absorbing area. Using this strategy there is no risk to create a source term while the desired decay of the wavefunction should be still achieved via the kinetic operator term.

In order to test our expectations we have repeated the simulations by dropping the term $\exp (i \eta)$ in Eq. (8) and $\exp (-i \eta)$ in Eq. (9), the results are shown in Fig. 3, The comparison with the respective panels of Fig. 3] show immediately that the desired effect is achieved. In particular in the low-frequency case (right hand column) the wavefunction is absorbed at the edges of the grid and no signature of explosion is seen anymore.

We may therefore conclude that the exterior complex scaling technique can be applied as an absorbing boundary to time-dependent simulations on laser-atom simulations in the length as well as in the velocity gauge as long as the complex factors in Eqs. (8) and (9) are removed. We may note that McCurdy et al. [33] reached to a similar conclusion for the velocity gauge as they did not transform the momentum $\hat{p}_{Z}$ to the complex plane. Our analysis above shows that an analogous restriction is possible in the length gauge as well. Our test calculations have shown that, in general, after omission of the unstable factors calculations in the length gauge show slightly better results than those in the velocity gauge. We therefore restrict ourselves below to the length gauge only.

Thus, the above implementation of the ECS technique coincides with the desired absorbing boundary condition in time-dependent strong-field calculations. It has been shown in the application of ECS to the time-independent Schrödinger equation (e.g. [35, 38] and for review [24]) that using a sharp exterior scaling the derivative discontinuity at the boundary is handled exactly, as long as the boundary is chosen to coincide with a grid point. We have adapted this strategy in the time-dependent calculations. In test calculations we have found that the absorption effect at the boundaries is almost independent of the scaling angle $\eta$, in the present calculations we have used $\eta=\pi / 3$. Finally, before proceeding with a comparison of the ECS results with those obtained using the standard masking function technique we may note that special care has to be taken in the representation of the first and second derivatives in the transformed Schrödinger equation (for a detailed discussion, see [24]), which we have approximated using Lagrange interpolating polynomials [43].

\section{Comparison of ECS and masking function techniques as absorber}

In order to analyze the efficiency of the ECS method as absorber in time-dependent simulations as compared to the masking function technique we present in this section results of calculations for the interaction of the hydrogen atom with a linearly polarized laser field. We compare the results obtained using the ECS method with those obtained using conventional masking functions of the form:

$$
M=\cos ^{\frac{1}{8}}\left(\frac{\left|X-x_{i}\right|}{d} \frac{\pi}{2}\right),
$$

where $d$ is the length of the absorbing region, over which $M$ changes smoothly from 1 to 0 , and $x_{i}$ is the boundary point. Such a function has been applied at all boundaries of the numerical grid (c.f. gray zones in Fig. 11). In the course of the calculations we have tested different 

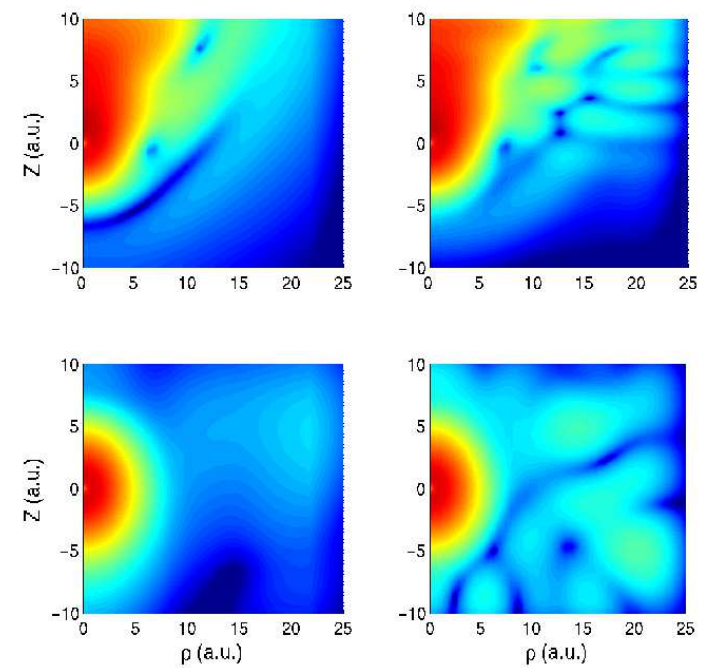

FIG. 4: (color online) Logarithmic contour plots of the electron density distributions from the numerical solution of the 2D TDSE of the hydrogen atom exposed to an intense laser pulse at $t=165$ a.u. (upper row) and $t=330$ a.u. (lower row). Results obtained using ECS (left hand panels) are compared with those obtained using the masking function (right hand panels) as absorber. Field parameters as in Eq. (12).

masking functions, the results presented below are found to be rather insensitive on the form of masking functions.

We use the time-dependent Schrödinger equation in the length gauge and the Coulomb potential of the hydrogen atom,

$$
V(z, \rho)=E(t) z-\frac{1}{\sqrt{z^{2}+\rho^{2}}} .
$$

The electric field profile is given by Eq. (12). The field parameters are the same as before. The grid parameters are $\Delta z=0.1$ a.u., $\Delta \rho=0.2$ a.u. and the time step $\Delta t=$ 0.1 a.u.. The initial ground states have been obtained via imaginary time propagation [44]. The absorber is applied at $\rho_{0}=22$ and $z_{1}=-z_{2}=-10$. The width of the absorber is chosen to be $20 \%$ of the grid size.

To demonstrate that the reduction of reflections is present in the solution of the TDSE, we present in Fig. 4 probability density distributions. From the comparison between the results for ECS (left column) and for the masking function (right column) at $t=165$ a.u. and $t=330$ a.u. in the upper and lower row, respectively, the difference in the efficiency of the two absorbers is clearly visible. The distributions obtained with the masking function show interference patterns due to the reflections at the boundaries, which are not seen in the results for the ECS absorber. Note that at $t=165$ a.u. the wave packet has reached (and is reflected from) the upper boundary in $Z$-direction only, while at the end of the pulse reflections in all directions have appeared.
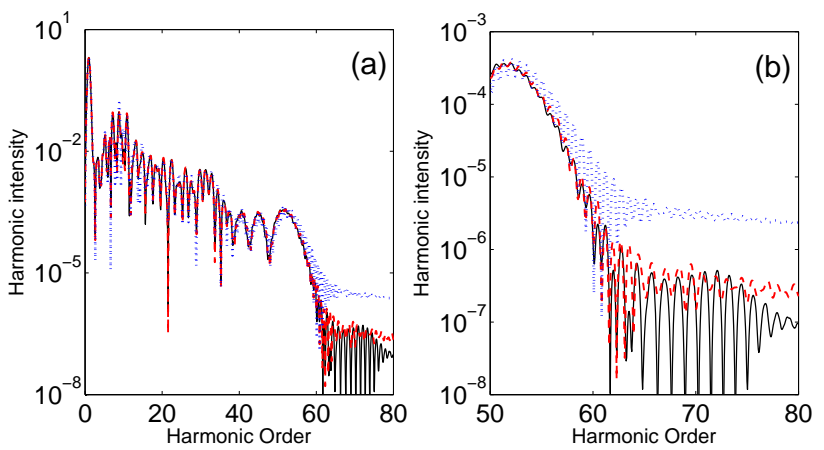

FIG. 5: (color online) High harmonic spectra from the interaction of the hydrogen atom with an intense linearly polarized 3-cycle laser pulse at $E_{0}=0.1$ a.u. and $\omega=0.057$ a.u.. Shown is a comparison between the results obtained from the (unrestricted) reference calculation (black solid line) and calculations using the ECS (red dashed line) and the masking function (blue dotted line) as absorbers at $z_{1}=-z_{2}=-80$ a.u.. In transversal direction the grid was large enough to avoid additional reflections from this boundary. (a) Full spectrum and (b) cut-off region.

\section{CALCULATION OF HIGH HARMONIC SPECTRA}

Finally, we apply the ECS absorber to a typical intense-field phenomenon, namely the evaluation of high harmonic spectra. High harmonic generation (HHG) is an important process for laser frequency conversion and the generation of attosecond pulses (for reviews, see e.g. 45, 46]). According to the semiclassical three-step rescattering picture [47, 48], and confirmed by quantummechanical calculations [49], HHG can be understood as the ionization of an electron by tunneling through the barrier of the combined Coulomb and laser fields, followed by the acceleration of the electron in the field, which may cause, for linear polarization of the field, a return of the electron to and its recombination with the parent ion under the emission of a harmonic photon. On the basis of this picture it is reasonable to limit the grid size of an ab-initio calculation of high harmonic spectra via the time-dependent Schrödinger equation, since beyond a certain distance from the nucleus outgoing wave packets are expected to have no effect on the high harmonic spectra.

We have performed simulations of the 2D TDSE for the hydrogen atom in an intense linearly polarized laser pulse, given by the pulse form in Eq. (12) with $E_{0}=0.1$ a.u. and $\omega=0.057$ a.u., and evaluated high harmonic spectra as the Fourier transform of the time-dependent dipole moment. Note that the dipole moment has been determined over the interior box (i.e. without the absorbing regions). In order to analyze the effect of reflections from the edges of the grid along the polarization 
direction on the spectra, we compare in Fig. 5 the results of three simulations, namely the full calculation as a reference (black solid line), performed on a sufficiently large simulation box to prevent reflections at the boundaries, and calculations using ECS (red dashed line) and masking function (blue dotted line) as absorbers. The calculations with absorbers have been restricted along the polarization axis by choosing $z_{1}=-z_{2}=-80$ a.u., which exceeds the maximum excursion of the classical electron trajectories of 63.6 a.u.. The absorbing part of the grid has been chosen to extend over an additional 20 a.u. at both ends. In the transversal direction the grid was chosen large enough to avoid reflections from this boundary.

The results in Fig. [5 $\mathrm{a}$ ) show the typical high harmonic spectrum with a plateau and a cut-off at $N=$ $\left(I_{p}+3.17 U_{p}\right) / \omega \approx 51$, where $I_{p}=0.5$ is the ionization potential of the hydrogen atom and $U_{p}=I_{0} / 4 \omega^{2}=0.77$ is the ponderomotive potential. From the comparison in Fig. 5 it is seen that the harmonics in the plateau do not differ significantly. There are small deviations in the minima between the harmonics obtained from the simulation with the masking function but the maxima appear to be unchanged. The effects of the reflections become visible at and beyond the cut-off, this region is enlarged in Fig. 5b). While the results from the ECS calculation almost agree with those from the full calculation over a decrease in the signal of two orders of magnitude, the results evaluated with the masking function start to differ near the cut-off and the deviations increase up to an order of magnitude in the signal beyond the cut-off. This "artificial" increase of the HHG signal in the cut-off region results from those parts of the wavefunction reflected at the boundary, which return to the nucleus and give rise to harmonics without physical meaning. Note that an accurate calculation of the harmonics in the cut-off regime is e.g. important for analysis of the generation of single attosecond pulses (e.g. [50]).

The effect of the reflections even increases for longer pulses, in which several wave packets reach the boundaries. This is seen from the results, presented in Fig. 6, where harmonic spectra obtained for laser fields with a constant envelope, $E=E_{0} \sin (\omega t)$ with $E_{0}=0.05$ and $\omega=0.057$, having 3 (upper row), 6 (middle row) and 12 (bottom row) cycles are shown. Results obtained using ECS and masking function as absorber techniques are shown in the left and right hand panels, respectively. The absorbers were placed at $z_{1}=-z_{2}=-35 \mathrm{a}$.u. (here the maximum excursion length is 30.8 a.u.), with an additional absorbing region of 6 a.u. The grid size in $\xi$ direction was big enough not to influence the results. While the results obtained for the 3-cycle field show the expected cut-off at the 19th harmonic, spurious harmonics due to reflections appear beyond the cut-off and increase in signal as the number of field cycles increases. The comparison shows that for the ECS results the contrast ratio between the false and the plateau harmonics is about $10^{-3}$ in all cases, while for the masking functions
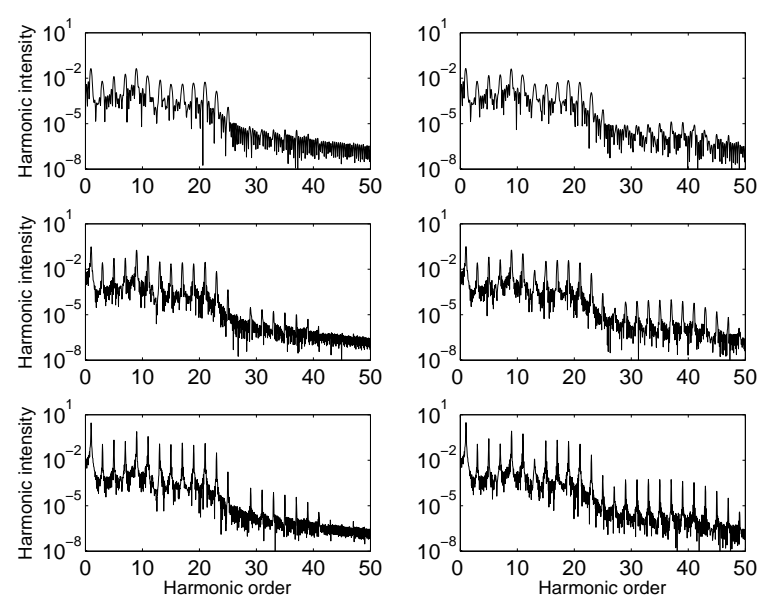

FIG. 6: Comparison of harmonic spectra calculated using the ECS (left column) and the masking function (right column) technique in laser pulse with constant amplitude having 3 (upper row), 6 (middle row) and 12 (lower row) cycles.

it increases, especially for the highest false harmonics, giving the impression of a second unphysical plateau.

\section{SUMMARY}

In summary, we have investigated the implementation of the exterior complex scaling technique as an absorber in the numerical solution of the time-dependent Schrödinger equation for strong-field problems on a grid. Our analysis has shown that the ECS technique can be applied in both the length and the velocity gauge as long as the untransformed field coupling is used on the entire grid including the absorbing area. It is found that the decay due to the ECS transformation in the kinetic operator term is sufficient to efficiently reduce reflections at the grid boundaries. A comparative study has shown that in this implementation a significantly better suppression of reflections can be achieved as using the conventional masking function method. By application of the ECS method to the evaluation of high harmonic spectra differences in the suppression of artifacts, e.g. in form of spurious harmonics, is demonstrated. The simple test cases considered here should capture the essence of the reflection problem, exterior complex scaling in both length and velocity gauge can be therefore considered as an efficient absorption technique for numerical time-dependent solutions in higher dimensions and/or of more complex processes too.

\section{Acknowledgment}

We thank S. Baier, P. Panek, L. Plaja, A. Requate, and J. R. Vázquez de Aldana for many stimulating discus- 
sions. This work has been partially supported by DAAD via project $\mathrm{D} / 05 / 25690$.
[1] D. Strickland and G. A. Mourou, Opt. Commun. 56, 219 (1985).

[2] G. A. Mourou, C. P. J. Barty, and M. D. Perry, Phys. Today 51, 22 (1998).

[3] F. H. M. Faisal, Theory of Multiphoton Processes (Plenum Press, New York, 1987).

[4] K. C. Kulander, Phys. Rev. A 35, 445 (1987).

[5] J. L. Krause, K. J. Schafer, and K. C. Kulander, Phys. Rev. A 45, 4998 (1992).

[6] H. G. Muller, Phys. Rev. A 60, 1341 (1999).

[7] M. Nurhuda and F. H. M. Faisal, Phys. Rev. A 60, 3125 (1999).

[8] R. Shakeshaft, R. M. Potvliege, M. Dörr, and W. E. Cocke, Phys. Rev. A 42, 1656 (1990).

[9] P. G. Burke, P. Franken, and C. J. Joachain, J. Phys. B 24, 761 (1991).

[10] L. Dimou and F. H. M. Faisal, Phys. Lett. A 171, 211 (1992).

[11] X. Tang, H. Rudolph, and P. Lambropoulos, Phys. Rev. Lett. 65, 3269 (1990)

[12] Ph. Antoine, B. Piraux, and A. Maquet, Phys. Rev. A 51, R1750 (1995).

[13] E. Cormier and P. Lambropoulos, J. Phys. B 29, 1667 (1996).

[14] L. V. Keldysh, Zh. Eksp. Teor. Fiz. 47, 1945 (1964) [Sov. Phys. JETP 20, 1307 (1965)].

[15] F. H. M. Faisal, J. Phys. B 6, L89 (1973).

[16] H. R. Reiss, Phys. Rev. A 22, 1786 (1980).

[17] A. Becker and F. H. M. Faisal, J. Phys. B 38, R1 (2005).

[18] K. Harumiya, H. Kono, Y. Fujimura, I. Kawata, and A. D. Bandrauk, Phys. Rev. A 66, 043403 (2002).

[19] S. Baier, C. Ruiz, L. Plaja, and A. Becker, Phys. Rev. A 71, 1 (2006)

[20] R. Santra and L. S. Cederbaum, Phys. Rep. 368, 1 (2002).

[21] J. G. Muga, J. P. Palao, B. Navarro, and I. L. Equsquiza, Phys. Rep. 395, 357 (2004).

[22] S. Yoshida, S. Watanabe, C. O. Reinhold, and J. Burgdörfer, Phys. Rev. A 60, 1113 (1999).

[23] Y. K. Ho, Phys. Rep. 99, 1 (1983)

[24] C. W. McCurdy, M Baertschy, and T. N. Rescigno, J. Phys. B 37, R137 (2004).

[25] R. Kosloff and D. Kosloff, J. Comput. Phys. 63, 363 (1986).

[26] K. Ishikawa and K. Midorikawa, Phys. Rev. A 65, 043405
(2002).

[27] K. Ishikawa, Phys. Rev. Lett. 91, 043002 (2003).

[28] R. Santra and C. H. Greene, Phys. Rev. A 70, 053401 (2004).

[29] A. Gordon, C. Jirauschek, and F. X. Kärtner, Phys. Rev. A 73, 042505 (2006).

[30] C. A. Nicolaides and D. R. Beck, Phys. Lett. A 65, 11 (1978).

[31] B. Simon, Phys. Lett. A 71, 211 (1979).

[32] C. W. McCurdy and C. K. Stroud, Comp. Phys. Comm. 63, 323 (1991)

[33] C. W. McCurdy, C. K. Stroud, and M. K. Wisinski Phys. Rev. A 43, 5980 (1991)

[34] Phys. Rev. A 65, 042714 (2002)

[35] T. N. Rescigno, M. Baertschy, W. A. Isaacs, and C. W. McCurdy, Science 286, 2474 (1999).

[36] T. N. Rescigno and C. W. McCurdy, Phys. Rev. A 62, 032706 (2000).

[37] I. Gilary, A. Fleischer, and N. Moiseyev, Phys. Rev. A 72, 012117 (2005).

[38] C. W. McCurdy, D. A. Horner, T. N. Rescigno, and F. Martín, Phys. Rev. A 69, 032707 (2004).

[39] W. Reinhardt, Ann. Rev. Phys. Chem. 33, 223 (1982).

[40] T. Regge, Nuovo Cimento 14, 951 (1959).

[41] G. Doolen, J. Nuttall, and R. Stagat, Phys. Rev. A 10, 1612 (1974).

[42] T. N. Rescigno and V. McKoy, Phys. Rev. A 12, 522 (1975).

[43] http://mathworld.wolfram.com/LagrangeInterpolatingPolynomial.ht

[44] R. Kosloff and H. Tal-ezer, Chem. Phys. Lett. 127, 223 (1986).

[45] T. Brabec and F. Krausz, Rev. Mod. Phys. 72, 545 (2000).

[46] P. Agostini and L. F. DiMauro, Rep. Prog. Phys. 67, 813 (2004).

[47] K. J. Schafer, B. Yang, L. F. DiMauro, and K. C. Kulander, Phys. Rev. Lett. 70, 1599 (1993).

[48] P. B. Corkum, Phys. Rev. Lett. ,71, 1994 (1993).

[49] M. Lewenstein, Ph. Balcou, M. Yu. Ivanov, A. L'Huillier, and P. B. Corkum, Phys. Rev. A , 49, 2117 (1994)

[50] M. Hentschel, R. Kienberger, Ch. Spielmann, G. A. Reider, N. Milosevic, T. Brabec, P. Corkum, U. Heinzmann, M. Drescher, and F. Krausz, Nature (London) 414, 661 (2000). 